\title{
Variation of the extinction law in the Trifid nebula
}

\author{
L. Cambrésy ${ }^{1}$, J. Rho ${ }^{2,4}$, D. J. Marshall ${ }^{3}$, and W. T. Reach ${ }^{2,4}$ \\ 1 Observatoire astronomique de Strasbourg, Université de Strasbourg, CNRS, UMR 7550, 11 rue de l’Université, 67000 Strasbourg, \\ France \\ e-mail: cambresy@astro.unistra.fr \\ 2 Infrared Processing and Analysis Center, California Institute of Technology, Pasadena, CA 91125, USA \\ 3 Institut de Recherche en Astrophysique et Planétologie de Toulouse (IRAP), Université Paul Sabatier, CNRS, UMR 7095, \\ 9 avenue du colonel Roche, 31028 Toulouse Cedex 4, France \\ e-mail: Douglas. Marshall@cesr.fr \\ 4 Stratospheric Observatory for Infrared Astronomy, Universities Space Research Association, Mail Stop 211-3, Moffett Field, \\ CA 94035, USA \\ e-mail: [jrho;wreach]@sofia.usra.edu
}

Received 4 October 2010 / Accepted 21 December 2010

\section{ABSTRACT}

\begin{abstract}
Context. In the past few years, the extinction law has been measured in the infrared wavelengths for various molecular clouds and different laws have been obtained.

Aims. In this paper we seek variations of the extinction law within the Trifid nebula region. Such variations would demonstrate local dust evolution linked to variation of the environment parameters such as the density or the interstellar radiation field.

Methods. The extinction values, $A_{\lambda} / A_{V}$, are obtained using the 2MASS, UKIDSS and Spitzer/GLIMPSE surveys. The technique is to inter-calibrate color-excess maps from different wavelengths to derive the extinction law and to map the extinction in the Trifid region.

Results. We measured the extinction law at 3.6, 4.5, and $5.8 \mu \mathrm{m}$ and we found a transition at $A_{V} \approx 20$ mag. Below this threshold the extinction law is as expected from models for $R_{V}=5.5$ whereas above 20 mag of visual extinction, it is flatter. Using these results the color-excess maps are converted into a composite extinction map of the Trifid nebula at a spatial resolution of 1 arcmin. A tridimensional analysis along the line-of-sight allowed us to estimate a distance of $2.7 \pm 0.5 \mathrm{kpc}$ for the Trifid. The comparison of the extinction with the $1.25 \mathrm{~mm}$ emission suggests the millimeter emissivity is enhanced in the dense condensations of the cloud. Conclusions. Our results suggest a dust transition at large extinction which has not been reported so far as well as dust emissivity variations.
\end{abstract}

Key words. dust, extinction - ISM: individual object: Trifid nebula - infrared: ISM - radio continuum: ISM

\section{Introduction}

During the last three decades, dust evolution in the interstellar medium (ISM) has been studied mostly through the grain emission in the far-infrared and then the sub-millimeter wavelengths. The analysis of the surface brightnesses at 60 and $100 \mu \mathrm{m}$ observed by IRAS revealed a small and a big grain component in the ISM where the small grains are the major contributor at $60 \mu \mathrm{m}$ (Boulanger et al. 1988; Laureijs et al. 1991). The transition from small to big grains occurs at a typical visual extinction of $1 \mathrm{mag}$. More recently a new transition was discovered in the dust composition with the formation of fluffy grains. The analysis of PRONAOS sub-millimeter observations by Bernard et al. (1999) resulted in the discovery of a population of enhanced-emissivity dust grains which have a lower equilibrium temperature. This emissivity enhancement in the farinfrared by a factor of about 3 (Cambrésy et al. 2001) requires the existence of composite grains formed by the coagulation of small grains. A large scale study on the whole Galactic anticenter hemisphere showed this transition also occurs at low extinction, $A_{V} \approx 1 \mathrm{mag}$ (Cambrésy et al. 2005). It might be closely related to the recent discovery made by Molinari et al. (2010a) with Herschel data where this same density threshold seems to indicate the transition where diffuse clouds start to collapse into filaments.

With the wealth of available deep data in the near and midinfrared it is now possible to investigate the interstellar grain properties at shorter wavelengths. In this spectral domain a lot of progresses has been made in the past few years through the study of the extinction law. Dense clouds can be probed on large scales and for various astrophysical environments. Recent studies with Spitzer data (Indebetouw et al. 2005; Flaherty et al. 2007; Román-Zúñiga et al. 2007; Chapman et al. 2009) focused on different clouds and obtained different extinction laws. We propose in this work to look for possible variations of the extinction curve within a single cloud. We chose an active star-forming region which includes the famous HII region M20, the Trifid nebula. Its location in the Galactic plane at only 7 degrees from the Galactic center offers a high stellar density which is ideal for probing the extinction distribution. The distance of this young nebula $\left(10^{5}\right.$ years $)$ is still debated. The observation of the central O7 star responsible for the photoionization, HD 164492, yields a distance ranging from $1.67 \mathrm{kpc}$ (Lynds et al. 1985) to $2.8 \mathrm{kpc}$ (Kohoutek et al. 1999). This active region has its first generation of massive stars interacting with the ISM and is very likely 
triggering a second generation of star formation (Cernicharo et al. 1998). The state-of-the-art for the Trifid star-forming region is presented in a review paper by Rho et al. (2008).

The extinction law is generally estimated in molecular clouds as a whole and the discrepancies between the different results are interpreted as variations of the dust optical properties from cloud-to-cloud due to the environment. Obviously variations should also be observed within molecular clouds. As the density increases from the envelope to the core of a cloud, the dust properties, hence the extinction law, is expected to be affected. An attempt to detect such a variation was proposed by Román-Zúñiga et al. (2007) in their study of a dark cloud core, B59, up to $A_{V} \approx 59 \mathrm{mag}$ but they did not succeed. Olofsson \& Olofsson (2010) used five different methods to derive the extinction law in B335. The scatter in their results was consistent with the uncertainties so they could not conclude on any evolution of the extinction law within the cloud. Chapman et al. (2009) managed to find variations by averaging their results from several clouds and they showed evidence for grain growth at $A_{K_{\mathrm{s}}}>1 \mathrm{mag}\left(A_{V}>9\right)$. A global galactocentric variation of the extinction law has also been reported by Zasowski et al. (2009) in a large scale study that excluded molecular clouds.

The present study focuses on the variations of the extinction law within the molecular cloud associated with the Trifid nebula. Our analysis relies on the 2MASS (Skrutskie et al. 2006), UKIDSS (Lawrence et al. 2007) and GLIMPSE II (Churchwell et al. 2009) surveys. Special attention is given to the data filtering to limit the possible biases as explained in Sect. 2. Our original method to derive the extinction law from 3.6 to $5.8 \mu \mathrm{m}$ is based on the color mapping rather than the classical analysis of the source list distribution in a color-color diagram. The details and the advantages of this technique are presented in Sect. 3 while Sect. 4 describes the final extinction map making and a tridimensional analysis of the Trifid line-of-sight which allows us to disentangle the complexity along this direction. In addition we studied the dust emissivity at $1.25 \mathrm{~mm}$ by comparing the masses derived from the dust continuum emission and the dust absorption. We conclude in Sect. 5.

\section{Data preparation}

\subsection{Datasets}

Our study covers a large field of $1.6^{\circ} \times 1.4^{\circ}$. The 2MASS nearinfrared data provides $J H K_{\mathrm{s}}$ fluxes for the whole sky. The high stellar density in the Trifid direction induces a loss of sensitivity as 2MASS sources with less than $5^{\prime \prime}$ separation are affected by confusion. The degradation reaches almost 1.5 mag in each filter, with completeness level of only $12.8 \mathrm{mag}$ for the $K_{\mathrm{s}}$ band for instance.

Deeper $J H K_{\mathrm{S}}$ fluxes are obtained from the UKIDSS 6th data release (UKIDSSDR6plus) of the Galactic Plane survey. UKIDSS uses the UKIRT Wide Field Camera (WFCAM, Casali et al. 2007). The photometric system is described in Hewett et al. (2006), and the calibration is described in Hodgkin et al. (2009). The pipeline processing and science archive are described in Hambly et al. (2008).

Longer wavelengths from Spitzer/IRAC are publicly available through the GLIMPSE II legacy survey. We used the high reliability point source Catalog (v2.1) which includes data from epochs 1 and 2 .

The merged catalog between 2MASS, UKIDSS and GLIMPSE tables results from a 1 " cone search. Completeness limit magnitudes for all filters are presented in Table 1. For bright
Table 1. Completeness limit magnitudes.

\begin{tabular}{ccccc}
\hline \hline & 2MASS & UKIDSS & UKIDSS(peak) & GLIMPSE \\
\hline$J$ & 14.4 & 16.2 & 17.8 & \\
$H$ & 13.5 & 15.0 & 16.7 & \\
$K_{\mathrm{s}}$ & 12.8 & 14.2 & 16.0 & \\
{$[3.6]$} & & & & 13.5 \\
{$[4.5]$} & & & & 13.4 \\
{$[5.8]$} & & & & 12.2 \\
{$[8.0]$} & & & & 11.4 \\
\hline
\end{tabular}

Notes. For UKIDSS the luminosity function slopes are not well defined, so the peak values of the function for sources with an error smaller than 0.15 mag are displayed.

stars, the 2MASS photometry is chosen over the UKIDSS photometry which is affected by saturation. Although the UKIDSS photometry is directly calibrated on 2MASS we found a systematic difference between the two systems:

$$
\begin{aligned}
J(2 \mathrm{MASS}) & =J(\mathrm{UKIDSS})+0.084 \\
H(2 \mathrm{MASS}) & =H(\mathrm{UKIDSS})-0.074 \\
K_{\mathrm{s}}(2 \mathrm{MASS}) & =K_{\mathrm{s}}(\mathrm{UKIDSS})-0.003 .
\end{aligned}
$$

We corrected the UKIDSS photometry to match the 2MASS system. The UKIDSS astrometry has also been corrected to match the GLIMPSE positions by adding 0.12 and 0.07 arcsec to the right ascension and declination, respectively. In the following, only magnitude measurements with an uncertainty smaller than 0.15 mag will be considered. It yields about $2 \times 10^{5}$ sources at $K_{\mathrm{s}}, 6 \times 10^{5}$ at $3.6 \mu \mathrm{m}$ in the range RA $\in[269.50,271.29]$ and Dec $\in[-23.6,-22.2]$.

\subsection{Source selection criteria}

The analysis of the stellar color excess used to investigate the cloud properties requires all stars to be background stars. The contamination by foreground objects cannot be neglected for the Trifid nebula located between 1.7 and $2.8 \mathrm{kpc}$ probably in the Scutum Arm, behind the Sagittarius Arm. The procedure to remove foreground stars is statistical for two reasons: 1) the number of detected sources is close to $6 \times 10^{5}$; and 2) there is a degeneracy between intrinsically red foreground objects and blue background sources. A color criteria would therefore be inefficient. It would actually lead to a source spatial distribution correlated with the dust distribution which is not consistent with the supposedly foreground nature of the stars. The individual identification is generally impossible, except in the high extinction region where the degeneracy can be raised. Keeping these constraints in mind, we choose a two step strategy, the first purely statistical and the second manually applied to the individual stars.

The statistical step consists in sampling the source catalog into a data cube with two spatial dimensions and a third dimension being a color axis. Only one star per box is allowed. Basically, stars are sampled by a 4 arcmin grid step according to their celestial coordinates and the third axis allows to color-sort the stars within the same $16 \operatorname{arcmin}^{2}$ pixel so that there is only a single star per 3D-box. The first slice of the cube represents a map where each pixel contains the color of the bluest object of the stack. The following slices contain redder and redder color values. As the completeness limit of UKIDSS is not well defined we use only 2MASS and GLIMPSE for this statistical procedure. The variation of the stellar density makes the number of stars per 
pixel, and therefore the size of the stack, range from 85 to 387. For a $16 \operatorname{arcmin}^{2}$ pixel, it means the source density varies from $19 \times 10^{3}$ to $87 \times 10^{3} \mathrm{deg}^{-2}$. Once the cube is built, each plane from the bluest to the reddest is compared to a raw color map of the field, i.e. a non-calibrated extinction map. A correlation with the color map starts at the 38th slice, meaning the distribution of the $\sim 38$ bluest stars in all the $16 \operatorname{arcmin}^{2}$ pixels is not correlated with the dust distribution. They must be considered as foreground ob-

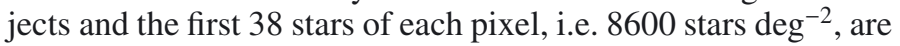
thus removed from the original catalog. This represents between $10 \%$ to $45 \%$ of the sources detected at $K_{\mathrm{s}}$ and [3.6] depending on the position, i.e. the local source density.

At this point, only a fraction of the foreground stars is removed. A second step is required to filter the catalog in the regions where the contamination is critical because the foreground objects may even be the dominant population. Fortunately, in these regions the color degeneracy is raised by the strong reddening from the dust grains. To define these regions we build a [3.6]-[4.5] color map following the adaptive method described in Sect. 3.1.1. Then we select the pixels where [3.6]-[4.5] > $0.35 \mathrm{mag}\left(A_{V} \approx 30 \mathrm{mag}\right)$ which yields a $25 \operatorname{arcmin}^{2}$ surface area. Such a high threshold guarantees that the degeneracy with the intrinsic star color is broken. For this manual operation the restriction on UKIDSS data can be waived and $\sim 10^{3}$ sources are detected in the 25 arcmin area at both $K_{\mathrm{s}}$ and $3.6 \mu \mathrm{m}$ with error smaller than $0.15 \mathrm{mag}$. The spectral energy distribution from $1.25 \mu \mathrm{m}$ to $8 \mu \mathrm{m}$ is examined for these sources. For each foreground star candidate, a color-composite $J H K_{\mathrm{s}}$ image is built to compare the color of the candidate itself with the color of the surrounding objects. This visual inspection allows us to perform the final classification of the source since a foreground star appears bluer than the surrounding objects. Among the $\sim 10^{3}$ sources detected at $K_{\mathrm{s}}$ and $3.6 \mu \mathrm{m}, 20 \%$ are classified as foreground stars. It corresponds to a total foreground star density of $\sim 37 \times 10^{3} \mathrm{deg}^{-2}$. It is worth noting that if we require sources to be detected at $J, H$ and $K_{\mathrm{s}}$ instead, the contamination level in the $25 \mathrm{arcmin}^{2}$ reaches $55 \%$. This is because many background stars are lost when the $J$ detection is required.

Beside the foreground stars, there is contamination by Young Stellar Objects (YSOs) that are formed in the molecular cloud associated with the Trifid nebula. Several studies, at various wavelengths, provide us with a source list of YSOs that we removed from our catalog. The most important contribution is from Rho et al. (2006) who analyzed the infrared colors from Spitzer IRAC and MIPS data. It yielded the identification of 161 YSOs (37 class I/O, 13 hot excess, 111 class II). Previous studies of the young star population in the Trifid nebula used 2MASS, ASCA, ROSAT (Rho et al. 2001) and Chandra (Rho et al. 2004).

Finally, another possible contamination would be extragalactic sources. This effect is negligible based on the location of the Trifid nebula in the Galactic plane and so close to the Galactic Center direction.

\section{Extinction law}

The general idea of the method to derive the extinction law is trivial. It requires the knowledge of the relative extinction in two wavelengths, $A_{\lambda 1} / A_{V} A_{\lambda 2} / A_{V}$, to evaluate the extinction at a third wavelength by measuring the slope of the star color distribution within a color-color plot $m_{\lambda 1}-m_{\lambda 2} / m_{\lambda 2}-m_{\lambda 3}$. As the color-color diagram slope is equal to the color-excess ratio $E_{\lambda 1-\lambda 2} / E_{\lambda 2-\lambda 3}$ the value of $A_{\lambda 3} / A_{V}$ is inferred. Practically, this procedure is not necessarily that simple because of the scatter in the color-color diagram. The selection of a sub-sample as clean

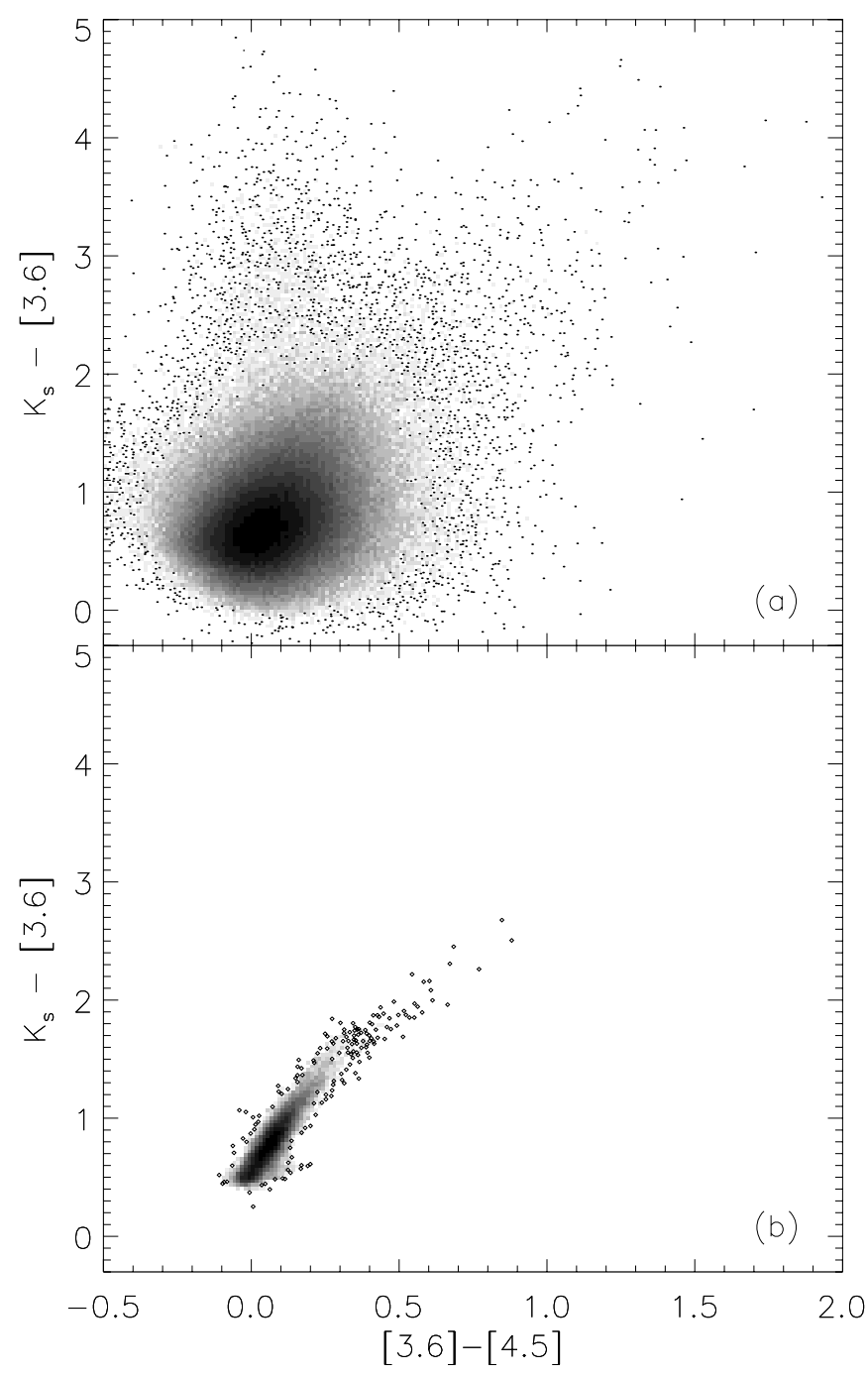

Fig. 1. a) Color-color diagram for stars with uncertainty smaller than 0.15 mag in the 3 bands. b) Color-color diagram obtained using color maps instead of the source list. The exact same stars are used for both plots. The gray scale represents the density of the data points in the plot and the dots show the individual data points at low density.

as possible is strongly recommended. Figure 1a is restricted to sources with an uncertainty smaller than $0.15 \mathrm{mag}$ at $K_{\mathrm{s}}, 3.6$ and $4.5 \mu \mathrm{m}$ although a large scatter is still present in the plot. In particular, the vertical elongation for [3.6] $-[4.5] \approx 0$ and $K_{\mathrm{S}}-[3.6]>2$ is not real. It is due to a severe underestimation of the UKIDSS $K_{\mathrm{s}}$ uncertainty for some sources. Fortunately, the method presented in the next section makes their contribution negligible because of their random spatial distribution.

Figure 1 and several others in this paper represent plots as density maps for clarity reason. Dark means high density. Unless specified otherwise the overlaid symbols simply help to visualize the lowest density pixels that are hardly visible.

\subsection{Color map making}

\subsubsection{Method}

Although a slope could be measured in Fig. 1a, the scatter is large. The Trifid line-of-sight crosses various ISM environments and many kind of stellar populations. This diversity, the high stellar density, and the relatively large distance of M 20 make our 
goals more difficult to reach than for nearby molecular clouds such as Chamaeleon, Taurus or Rho Ophiuchus.

We propose a method based on the color maps that spectacularly improves the accuracy of the analysis. Figure $1 \mathrm{~b}$ was built using the exact same sources as Fig. 1a and both figures have the same axis, same scale. While each point in Fig. 1a corresponds to a single source, points in Fig. 1b actually represent individual pixels taken from two color maps, one for each axis. Using the color allows us to keep the same axis as in Fig. 1a but it is obviously equivalent to an extinction map assuming an extinction law. The impressive difference between the two figures highlights the superiority of using maps rather than a source list directly. Using maps permits us to average the color of several individual nearby sources, and thus to reduce the scatter. Different methods are available to build an extinction map. Star count methods are sometimes used on large scale and for moderate extinction (Cambrésy 1999; Dobashi et al. 2005). This approach is definitely not adapted to a highly obscured distant cloud in the Galactic plane. The map would be noisy and seriously biased as it would be very sensitive to the foreground star contamination. Color-excess methods are better adapted. The first statistical method to map the extinction from color excess, called NICE, was proposed by Lada et al. (1994). Improvements were proposed and called NICER (Lombardi \& Alves 2001) and then NICEST (Lombardi 2009). A variant technique from Cambrésy et al. (2002) proposes to replace the arithmetic mean color by the median color in cells and to fix the number of sources within a cell rather than its size. The latter step makes the method adaptive since the spatial resolution is always optimal, adjusted to the local density. A final convolution can be applied if a fixed spatial resolution is wished. This convolution minimizes bias that would result in an underestimation of the extinction by using larger cells already at the final resolution size. Moreover the median is a powerful tool to limit the effect of the residual contamination by foreground stars and young stellar objects as detailed in Cambrésy et al. (2002).

All the color maps built for this work use the median color and follow the adaptive cell scheme with a fixed number of 3 stars per cell. The variable cell spatial resolution being $r_{i}$, a convolution by a Gaussian kernel of $\sqrt{1-r_{i}^{2}}$ arcmin is applied to reach a final resolution of 1 arcmin over the whole map.

\subsubsection{Selection bias}

Once a clean star sample is produced, i.e. filtered from foreground stars, YSOs and noisy photometry sources, the color maps are still subject to a selection bias. While it is mandatory to keep sources detected in all 3 bands to plot Fig. 1a, Fig. 1b needs two color maps that can be built independently with only a 2 band detection criteria for each of them. Figure 2 illustrates the color bias for [3.6] - [4.5] when it is obtained from two different sets of 3 band detection criteria, $K_{\mathrm{s}} /[3.6] /[4.5]$ or [3.6]/[4.5]/[5.8]. To exaggerate the effect $K_{\mathrm{s}}$ is taken from 2MASS which is not as deep as UKIDSS. The [3.6] - [4.5] average color is affected by the $K_{\mathrm{s}}$ detection criterion which makes the color bluer, and by the [5.8] detection criterion which tends to select redder objects. It is not a simple offset as shown in Fig. 2 because this effect is more important for redder color. Taking sources detected in the 3 bluer wavelengths yields an underestimation of the extinction. Using deeper $K_{\mathrm{s}}$ data would obviously help since more sources detected at [3.6] and [4.5] would also be seen at $K_{\mathrm{s}}$. However the effect cannot be avoided at large

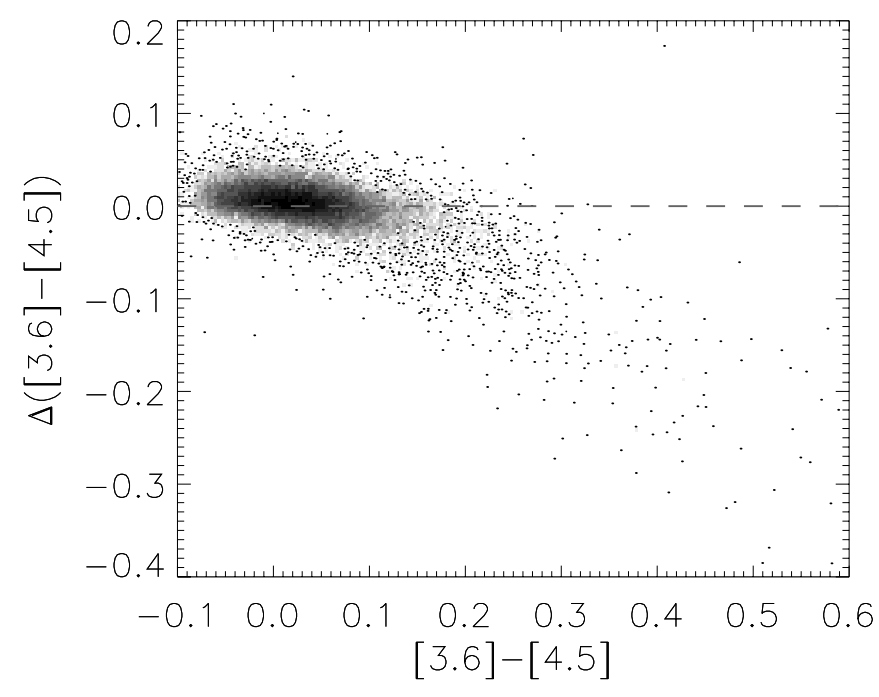

Fig. 2. [3.6] - [4.5] color difference from sources detected simultaneously in two different sets of 3 filters, $K_{\mathrm{s}}(2 \mathrm{MASS}) /[3.6] /[4.5]$ and [3.6]/[4.5]/[5.8], versus color.

extinction because the $K_{\mathrm{s}}$ band suffers from twice the extinction than the [3.6] one.

It is therefore critical to compare only maps that are built with the exact same sample of stars. It implies that the [3.6] [4.5] color map needs to be built from a source list which contains stars detected in the 3 bands $K_{\mathrm{s}}$, [3.6] and [4.5] if it is compared to a $K_{\mathrm{s}}-[3.6]$ map, or from a source list which contains stars detected at [3.6], [4.5] and [5.8] if it is compared to a [4.5] - [5.8] map.

A total of 6 color maps are built for our study: $H-K_{\mathrm{s}}$, $K_{\mathrm{s}}-$ [3.6], [3.6] - [4.5], [4.5] - [5.8]. Each map is obtained with sources detected in 3 bands at the same time. There are 2 versions for the $K_{\mathrm{s}}-$ [3.6] map and the [3.6] - [4.5] map because the third band can either be the bluer or the redder one.

\subsection{Results}

Although the extinction law has been considered as universal in the infrared for some time, it is now admitted that there are variations at wavelengths longer than $2 \mu \mathrm{m}$. The spectral range that is pseudo-universal is actually restricted to $1-2 \mu \mathrm{m}$ where the extinction is independent or only slightly dependent on the environment, i.e. dust temperature and density. Consequently, $A_{\mathrm{H}} / A_{V}=0.175$ and $A_{K_{\mathrm{s}}} / A_{V}=0.112$ (Rieke \& Lebofsky 1985) are assumed to be constant. This corresponds to $\beta=1.7$ in the expression $A_{\lambda} \propto \lambda^{-\beta}$ that is often used to describe the nearinfrared extinction law. As values from 1.6 to 1.8 can be considered reasonable, the effect of such a systematic uncertainty is discussed further in this section. The $H-K_{\mathrm{s}}$ map can be converted into an extinction map using

$$
A_{V}=\frac{\left(H-K_{\mathrm{s}}\right)-\left(H-K_{\mathrm{s}}\right)_{0}}{A_{\mathrm{H}} / A_{V}-A_{K_{\mathrm{s}}} / A_{V}}=15.87 E_{H-K_{\mathrm{s}}} .
$$

The extinction law is normalized to $A_{V}$ mainly for historical reasons but it is more convenient to normalize to $K_{\mathrm{s}}$. The relation between the extinction and the color excess becomes $A_{K_{\mathrm{s}}}=1.78 E_{H-K_{\mathrm{s}}}$. The reference color $\left(H-K_{\mathrm{s}}\right)_{0}=0.54 \mathrm{mag}$ is estimated from the area with the smallest values within the map, about 50 arcmin south-west from the densest core of the cloud. This is much redder than the intrinsic stellar color because it includes the diffuse extinction along the line-of-sight. 


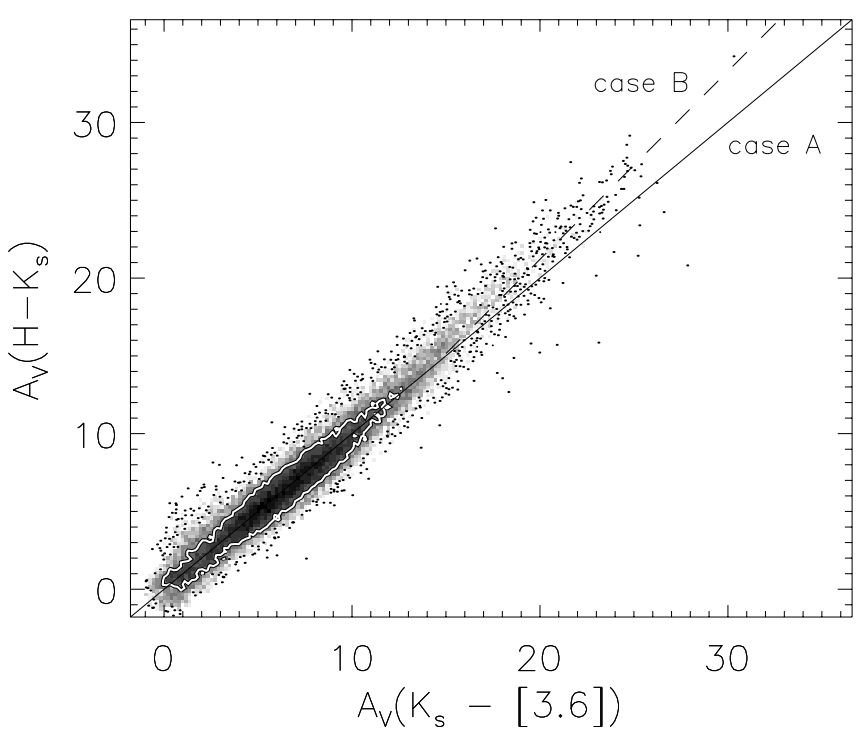

Fig. 3. Extinction from $H-K_{\mathrm{s}}$ color excess versus extinction from $K_{\mathrm{s}}-$ [3.6] color excess. By adjusting $K_{\mathrm{s}}-$ [3.6] to obtain a one-toone regression line (solid line) we find $A_{[3.6]} / A_{K_{\mathrm{s}}}=0.611$. The white iso-density contour delineates the region used to compute de regression line. The dashed line is the regression line for $A_{V}>20 \mathrm{mag}$, it indicates the deviation at large extinction. Case A and B refer to Table 2.

The reference color is necessary for providing an absolute extinction for the Trifid alone and it is discussed in the Sect. 4.2. It has absolutely no effect on the extinction law estimation nor on the relative extinction comparison from different parts of the field.

Figure 3 represents $A_{V}$ from $H-K_{\mathrm{s}}$ versus $A_{V}$ from $K_{\mathrm{S}}-$ [3.6]. Since $A_{V}=A_{K_{\mathrm{s}}} / 0.112$ is assumed, and by definition $A_{K_{\mathrm{s}}}=$ $E_{K_{\mathrm{s}}-[3.6]} /\left(1-A_{[3.6] / A_{K_{\mathrm{s}}}}\right)$, the only free parameter to adjust to obtain a slope of 1 is the value of $A_{[3.6]} / A_{K_{\mathrm{S}}}$. After the adjustment, it yields the value of $A_{[3.6]} / A_{K_{\mathrm{s}}}=0.611$. Figure 3 is presented as a density map for clarity and also because the density information is taken into account in the fitting process to exclude the low density pixels. Basically the minimum density level is fixed to $20 \%$ of the maximum density in the map. The color-coding used in the density plots is in log-scale so the $20 \%$ always corresponds to the dark region. The white iso-density contour delineates the region selected to compute the regression line in Fig. 3. Any outlier is therefore fully eliminated with this technique.

Once $A_{[3.6]} / A_{K_{\mathrm{s}}}$ is determined, $A_{[4.5]} / A_{K_{\mathrm{s}}}$ is evaluated with a plot of $A_{V}$ from $K_{\mathrm{s}}-$ [3.6] versus $A_{V}$ from [3.6] - [4.5]. A third similar plot will then provide $A_{[5.8]} / A_{K_{\mathrm{s}}}$ (see Fig. 4). The results are $A_{[4.5]} / A_{K_{\mathrm{s}}}=0.500$ and $A_{[5.8]} / A_{K_{\mathrm{s}}}=0.398$.

Since the pixels falling in the low density regions of the $A_{V}$ vs. $A_{V}$ plot are ignored the fitting is performed on regions where the visual extinction is roughly smaller than 15 mag. At large extinctions a striking deviation of the slope is observed and outlined by the dashed lines in Figs. 3 and 4 . The change in the slope occurs at $A_{V} \approx 20 \mathrm{mag}\left(A_{K_{\mathrm{s}}} \approx 2.2\right)$ and suggests a transition phase in the dust grain population. At such a high column density this result is unexpected. Contrary to studies in the far-infrared wavelengths which rely on the dust emission, there is no temperature degeneracy issue since it is based on stellar color mapping. A drawback of this technique is its limitation to the near-infrared spectral range. At wavelengths longer than $\sim 5 \mu \mathrm{m}$ the number of detected stars is too low to have an acceptable spatial sampling of the molecular clouds while shorter wavelengths are so sensitive to the extinction that the cloud's densest regions become totally opaque. A direct consequence of this limitation is that no evidence for a slope change can be seen in a $J-H$ vs. $H-K_{\mathrm{s}}$ diagram because the visual extinction cannot go deeper than $\sim 20$ mag with $J-H$. This prevents any conclusion on the extinction law variations at wavelengths shorter than $3.6 \mu \mathrm{m}$. Table 2 summarizes the results obtained from the slope measured in both Figs. 3 and 4. We consider two different cases for $A_{[3.6]} / A_{K_{\mathrm{s}}}$ at $A_{V}>20 \mathrm{mag}$ :

- case A: $A_{[3.6]} / A_{K_{\mathrm{s}}}=0.611$ is independent from the extinction, the value remains identical to lower extinctions (solid line in Fig. 3).

- case B: $A_{[3.6]} / A_{K_{\mathrm{s}}}=0.678$ which is the value actually obtained from the slope of the dashed line in Fig. 3.

The statistical errors on the slope estimations are small, as are the errors on the computed $A_{\lambda} / A_{K_{\mathrm{s}}}$. The systematic error on $A_{\mathrm{H}} / A_{K_{\mathrm{s}}}$ is the major source of uncertainty. We assume a value of 1.563 which corresponds to a power-law index of $\beta=1.7$ for the extinction law. Our results from Table 2 are plotted in Fig. 5. The shaded area delineates the systematic uncertainty for $\beta$ which varies from 1.6 to 1.8 . Using $\beta=1.6$, such as in Chapman et al. (2009), increases $A_{\lambda} / A_{K_{\mathrm{s}}}$ to the upper border of the shaded area. Nishiyama et al. (2006) reported a higher value of $\beta=1.99$ which is not compatible with our observations. The Weingartner \& Draine (2001, hereafter WD01) model is overlaid for comparison. The variations in the extinction law found in this work are rather small compared to the dust evolution from $R_{V}=3.1$ to $R_{V}=5.5$. The variation observed at visual extinction larger than 20 mag is likely the main reason that this dust transition is unknown. In case A the extinction parameters at 4.5 and $5.8 \mu \mathrm{m}$ are smaller at large extinction than at small extinction which is not realistic according the WD01 model. This case teaches us that the small deviation observed in Fig. 3 cannot be ignored as it would make longer wavelength results inconsistent. Case B is more likely even if it yields a larger value than reported in the literature until now. The largest value prior to this work was from Chapman et al. (2009) with $A_{[3.6]} / A_{K_{\mathrm{s}}}=0.64$. However their numbers are overestimated compared to ours or to those from Flaherty et al. (2007) because they chose a smaller $\beta$. Their results come from an average for Ophiuchus, Perseus and the Serpens molecular clouds which all form low-mass stars whereas the cloud associated with the Trifid is clearly more massive and forms OB stars. Flaherty et al. (2007) investigate the high-mass star-forming region of Orion and obtain a similar number of $0.636 \pm 0.003$, significantly below our $A_{[3.6]} / A_{K_{\mathrm{s}}}=0.678 \pm 0.006$. They did find smaller values at all wavelengths in low-mass star-forming regions. The presence of massive stars, in addition to the high density, may therefore be relevant.

WD01 attribute the variation of the extinction law with $R_{V}$ to grain growth only, although ice mantles onto dust grains contribute to the optical property variations. The main ice features relevant to our study originate from $\mathrm{H}_{2} \mathrm{O}$ and $\mathrm{CO}_{2}$ ices with absorption at $3.0 \mu \mathrm{m}$ and $4.27 \mu \mathrm{m}$, respectively. Both $\mathrm{H}_{2} \mathrm{O}$ and $\mathrm{CO}_{2}$ ices are detected at visual extinction below 4 mag (Whittet et al. 2001; Bergin et al. 2005) where the WD01 model is still valid. This indicates ice is not sufficient to significantly modify the extinction law in the Spitzer/IRAC filters. We can also rule out the possibility that ice features saturate at $A_{V} \approx 20 \mathrm{mag}$ because they are observed at larger extinctions without any saturation (Boogert, private communication). An example spectrum is presented in Whittet et al. (1998, Fig. 1) for a star with $A_{V}=$ 21 mag. It is worth noting the $\mathrm{H}_{2} \mathrm{O}$ ice band at $3.0 \mu \mathrm{m}$ peaks outside the [3.6] IRAC filter $(3.2-3.9 \mu \mathrm{m})$. Beside the absorption 

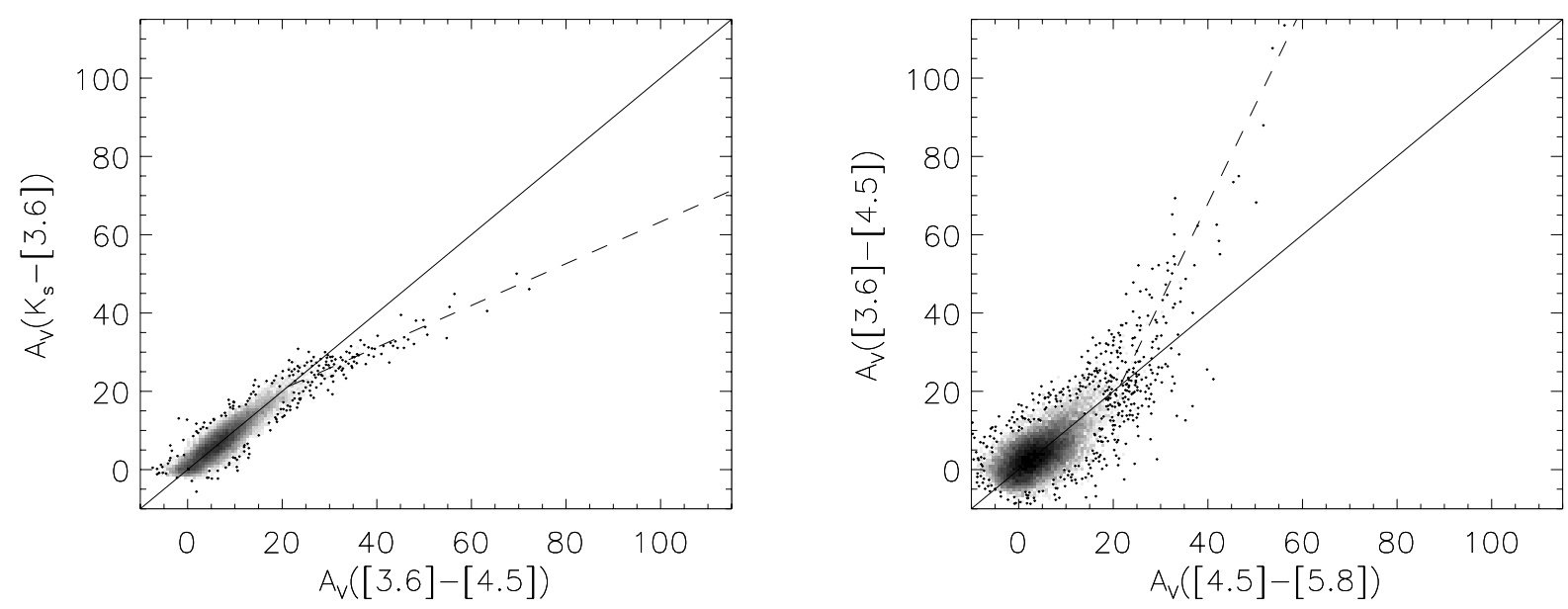

Fig. 4. Each plot represents the extinction correlation from two different color excesses. The two parameters $A_{[4.5]} / A_{K_{\mathrm{s}}}=0.500$ (left plot) and $A_{[5.8]} / A_{K_{\mathrm{s}}}=0.398$ (right plot) are adjusted to obtain a one-to-one regression line (solid line). The dashed lines trace the deviation at large extinction, the corresponding slopes in these plots are $0.55 \pm 0.02$ and $2.52 \pm 0.09$, respectively.

Table 2. Color excess ratios and resulting extinction law parameters.

\begin{tabular}{|c|c|c|c|c|c|c|c|}
\hline & $E_{H-K_{\mathrm{s}}} / E_{K_{\mathrm{s}}-[3.6]}$ & $E_{K_{\mathrm{S}}-[3.6]} / E_{[3.6]-[4.5]}$ & $E_{[3.6]-[4.5]} / E_{[4.5]-[5.8]}$ & $A_{\mathrm{H}} / A_{K_{\mathrm{s}}}$ & $A_{[3.6]} / A_{K_{\mathrm{s}}}$ & $A_{[4.5]} / A_{K_{\mathrm{s}}}$ & $A_{[5.8]} / A_{K_{\mathrm{s}}}$ \\
\hline$A_{V}<15$ & $1.446 \pm 0.002$ & $3.508 \pm 0.011$ & $1.087 \pm 0.007$ & 1.563 & $0.611 \pm 0.002$ & $0.500 \pm 0.003$ & $0.398 \pm 0.005$ \\
\hline \\
\hline case A & $1.446 \pm 0.002$ & $1.915 \pm 0.018$ & $1.496 \pm 0.089$ & 1.563 & $0.611 \pm 0.002$ & $0.408 \pm 0.003$ & $0.272 \pm 0.011$ \\
\hline case B & $1.749 \pm 0.003$ & $2.317 \pm 0.027$ & $1.810 \pm 0.091$ & 1.563 & $0.678 \pm 0.006$ & $0.540 \pm 0.003$ & $0.463 \pm 0.007$ \\
\hline case $\mathrm{B}, \beta=1.6$ & & & & 1.520 & $0.703 \pm 0.005$ & $0.573 \pm 0.003$ & $0.500 \pm 0.007$ \\
\hline case $\mathrm{B}, \beta=1.8$ & & & & 1.601 & $0.656 \pm 0.006$ & $0.507 \pm 0.003$ & $0.424 \pm 0.007$ \\
\hline \multicolumn{4}{|c|}{ Román-Zúñiga et al. (2007) for Barnard 59} & 1.55 & $0.619 \pm 0.029$ & $0.512 \pm 0.022$ & $0.459 \pm 0.024$ \\
\hline \multicolumn{4}{|c|}{ Flaherty et al. (2007) for Orion } & 1.55 & $0.636 \pm 0.003$ & $0.540 \pm 0.003$ & $0.504 \pm 0.003$ \\
\hline \multicolumn{4}{|c|}{ Chapman et al. (2009) for Ophiuchus, Perseus and Serpens } & 1.52 & $0.640 \pm 0.030$ & $0.530 \pm 0.030$ & $0.460 \pm 0.030$ \\
\hline \multicolumn{5}{|c|}{ Weingartner \& Draine (2001) with $R_{V}=3.1$} & 0.40 & 0.25 & 0.17 \\
\hline \multicolumn{5}{|c|}{ Weingartner \& Draine (2001) with $R_{V}=5.5$} & 0.60 & 0.49 & 0.40 \\
\hline
\end{tabular}

Notes. The two rows for $A_{V}>20$ mag represent different cases for $A_{[3.6]} / A_{K_{\mathrm{s}}}$ : case A propagates the same value as for small extinction, case B takes the actual measured value. We assume the power-law index $\beta=1.7$ (i.e. $A_{\mathrm{H}} / A_{K_{\mathrm{s}}}=1.563$ ) and results for $\beta=1.6$ and 1.8 are provided for case B. The main results from the literature for dense interstellar medium directions and from a dust model are indicated for comparison.

features, ice mantles make the grains more sticky, helping the coagulation process. Using Spitzer/IRS spectra McClure (2009) found evidence for a flattening of the extinction law at $A_{K}>$ 1 mag which would be due to the coagulation of ice-mantled grains. This suggests ice makes the coagulation process more efficient but it is not the dominant parameter since the coagulation starts well after ice-mantles appear. The abrupt change in the extinction law at $A_{V} \approx 20$ is not understood and we can only speculate that the high density in the Trifid cloud might allow a new structuring of the dust grains or that the shocks observed in the Trifid cloud could be determinant. In a recent study Boogert et al. (2011) also reported a flatter extinction law than WD01 based on a spectrum from 3 to $24 \mu \mathrm{m}$ for a star with 25 mag of visual extinction.

\section{From color to extinction}

\subsection{Composite map}

The extinction law parameters derived in the previous section allow us to convert the color maps into extinction. To proceed we follow the more realistic case $\mathrm{B}$ for $A_{\lambda} / A_{K_{\mathrm{s}}}$ when $A_{V}>20 \mathrm{mag}$ (see Table 2) and a reference color of $H-K_{\mathrm{s}}=0.54 \mathrm{mag}$. The associated zero points derived for the other colors are $K_{\mathrm{s}}-[3.6]=$ $0.497,[3.6]-[4.5]=-0.017$ and [4.5] $-[5.8]=0.187 \mathrm{mag}$. The sensitivity of each map relies on the wavelengths used to build the color maps. Shorter wavelengths are more sensitive to smaller extinction. The final extinction map presented in Fig. 6 is a combination defined as follow:

$$
A_{V} \text { from }\left\{\begin{array}{rll}
K_{\mathrm{s}}-[3.6] & \text { if } A_{V}<15 \mathrm{mag} \\
{[3.6]-[4.5]} & \text { if } 10<A_{V}<80 \mathrm{mag} \\
{[4.5]-[5.8]} & \text { if } \quad A_{V}>60 \mathrm{mag} .
\end{array}\right.
$$

The transition between two maps is progressive and linear within the overlapping extinction ranges. A maximum visual extinction of $\sim 80$ mag is reached. In complement to the extinction map Fig. 6 shows a close-up on the Trifid nebula observed at $8 \mu \mathrm{m}$ with the iso-extinction contours superimposed. The parent molecular cloud is an elongated structure with its densest region lying just West of the famous optical nebula. 


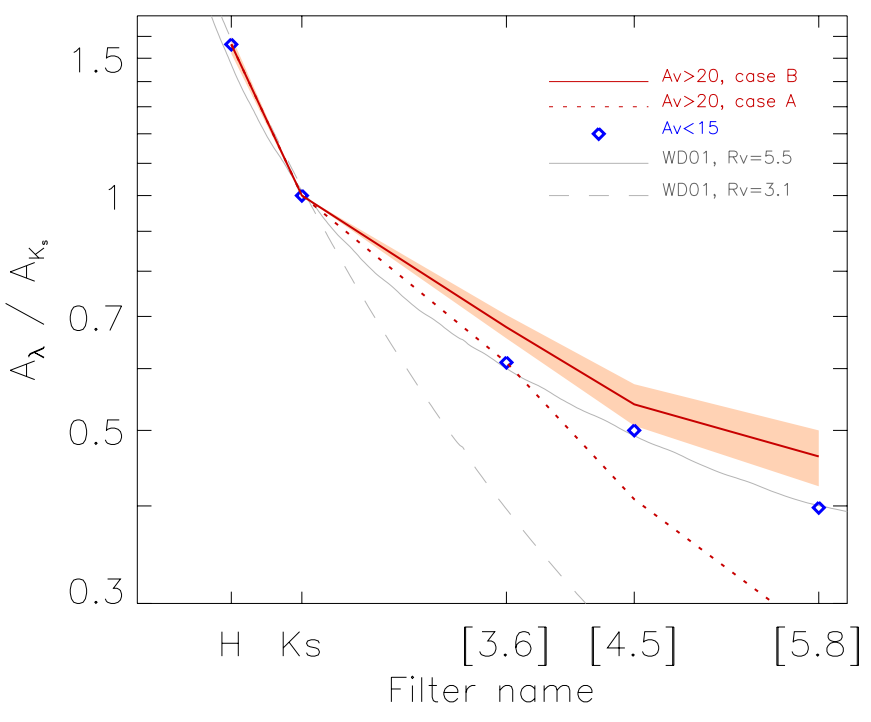

Fig. 5. Extinction law. The two red lines correspond to the cases described in Table 2 for $A_{V}>20 \mathrm{mag}$. The shaded area indicates the systematic error, for case $\mathrm{B}$ only, resulting from $A_{\mathrm{H}} / A_{K_{\mathrm{S}}}$ uncertainty which can range from $1.52(\beta=1.6)$ to $1.60(\beta=1.8)$. At longer wavelengths than $K_{\mathrm{s}}, \beta=1.6$ gives larger $A_{\lambda} / A_{K_{\mathrm{S}}}$. Diamonds are for $A_{V}<15 \mathrm{mag}$. The Weingartner \& Draine (2001) model is plotted in gray with dashed and plain lines for $R_{V}=3.1$ and $R_{V}=5.5$, respectively. The axis scale is linear for $x$ and logarithmic for $y$.

\subsection{Tridimensional analysis, distance estimation}

The extinction estimation relies on background stars. The meaning of background is not that trivial in the direction of the Trifid nebula since the line-of-sight crosses the whole galactic disk at only $1 \mathrm{kpc}$ from the Galactic center. The complexity of the lineof-sight and the distance of the stars used for the analysis are critical and can change our interpretation of the color maps. Even the distance of the nebula is still uncertain. To address these issues we performed a tridimensional analysis which aims to separate the different components along the line-of-sight.

A three dimensional stellar model of the Galaxy (Robin et al. 2003) is used to simulate the stellar distribution along the lineof-sight towards the Trifid nebula. The simulated stars are not subjected to extinction and so they provide an estimate of the intrinsic color and distance of the stars along this line-of-sight. By comparing the color distribution of the unreddened, simulated, stars to the observed stellar color distribution it is possible to infer the extinction distribution along the line-of-sight (Marshall et al. 2006). We use observations from 2MASS, UKIDSS and GLIMPSE and use the $K_{\mathrm{s}}-$ [3.6] color to probe the line-of-sight extinction. This technique is relatively independent of the stellar counts and reflects the changing stellar color as a function of distance (see Marshall et al. 2006, for more details). Furthermore there is no need to remove foreground stars as we are comparing the observations to a model where all stars are included. However, care needs to be taken to ensure that the model is indeed representative of the observations, so the simulated catalog is cut at the completeness magnitude of the observations in the $K_{\text {s }}$, [3.6] and [4.5] bands. Recently, Marshall et al. (2009) improved on this method using a genetic algorithm to deduce the line-of-sight extinction necessary to reproduce the observed stellar color distribution along a given line-of-sight. This version is the one we chose to use as it provides higher angular resolution ( $\sim 3$ arcmin compared to 15 arcmin previously) and more sensitivity to extinction features within $2 \mathrm{kpc}$ from the Sun. The resulting distances are subject to an uncertainty of roughly $15 \%$ but this may be higher at large distances and through high column densities. This results in a smearing out of extinction features along the line of sight, and could explain why, in Fig. 7, a narrow extinction peak is found at $6.5 \mathrm{kpc}$ when the extinction is low but this same peak is found to be wider through the higher density lines-of-sight crossing the Trifid nebula. At such a distance the interstellar material can be related to several Galactic structures which are the molecular ring (about 3-4 kpc from the Galactic center), the start of the Norma Arm, and the extremity of the Galactic bar.

The results of the 3D modeling is presented in Fig. 7. The extinction profile for the region where $A_{V}>20$ mag (see Fig. 6) provides us with a direct estimation of the cloud distance. Fitting a Gaussian yields a distance of $2.7 \pm 0.5 \mathrm{kpc}$. The extension of the cloud along the line-of-sight is likely from 50 to $100 \mathrm{pc}$ so the Gaussian standard deviation represents the uncertainty inherent to the 3D modeling, not the cloud size. The literature often refers to $1.7 \mathrm{kpc}$ (Lynds et al. 1985) for the Trifid nebula distance although a serious alternative is proposed by Kohoutek et al. (1999) with a distance between 2.5 and $2.8 \mathrm{kpc}$. Both estimations are based on the photometry of the central stars. The second work is more complete as it uses more stars and includes spectroscopy and accurate photometry. Our analysis of the tridimensional stellar distribution unambiguously supports the long distance hypothesis which place the Trifid in the Scutum Arm (Vallée 2008) rather than the Sagittarius Arm as generally reported. In the following we assume $d=2.7 \mathrm{kpc}$.

For such a distance, the foreground star number is expected to be at least $37 \times 10^{3} \mathrm{deg}^{-2}$ (see Fig. 7), or $11 \times 10^{3} \mathrm{deg}^{-2}$ if we restrict the sample to $2 \mathrm{MASS}$ sources. This is in good agreement with the analysis presented in Sect. 2.2 where we found a total foreground star density of at least $37 \times 10^{3} \mathrm{deg}^{-2}$ towards the dense region of the cloud and in agreement with the distribu-

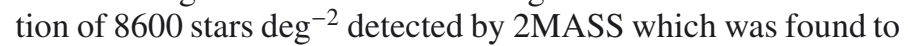
be uncorrelated with the extinction, i.e. foreground to the cloud. It worth noting the Sect. 2.2 results are also incompatible with the short distance hypothesis. The ${ }^{12} \mathrm{CO}$ velocity obtained from Dame et al. (2001) shows a velocity structure consistent with our extinction distribution which corroborates our estimation. The Trifid molecular clouds LSR velocity is about $18-20 \mathrm{~km} \mathrm{~s}^{-1}$. Assuming the Sun kinematics from McMillan \& Binney (2010), a distance to the Galactic center of $7.8 \mathrm{kpc}$ and a rotation speed of $247 \mathrm{~km} \mathrm{~s}^{-1}$ the Trifid velocity corresponds to a kinematic distance of 3.2-3.4 kpc which is even larger than our estimation based on the stellar population model.

The 3D analysis estimates the diffuse extinction in this direction to be $\sim 3$ mag $\mathrm{kpc}^{-1}$ and it shows that no other dense clouds significantly contaminate the Trifid line-of-sight. The stellar fraction at distances larger $8 \mathrm{kpc}$ represents only $7.7 \%$ of the background stars at large extinction and $2.6 \%$ at small extinction. In both cases their median distance is $\sim 5.8 \mathrm{kpc}$. The zero point chosen for the extinction map (Fig. 6) is validated by the $3 \mathrm{D}$ study as 1) there is no peak at $\sim 3 \mathrm{kpc}$ at low extinction and 2) it compensates for the diffuse extinction on the line-ofsight and the contamination at $6.5 \mathrm{kpc}$.

\subsection{Cloud mass}

The cloud mass is derived from the extinction map using the following relation (Dickman 1978):

$$
M=(\alpha d)^{2} \mu \frac{N_{\mathrm{H}}}{A_{V}} \sum_{i} A_{V}(i)
$$



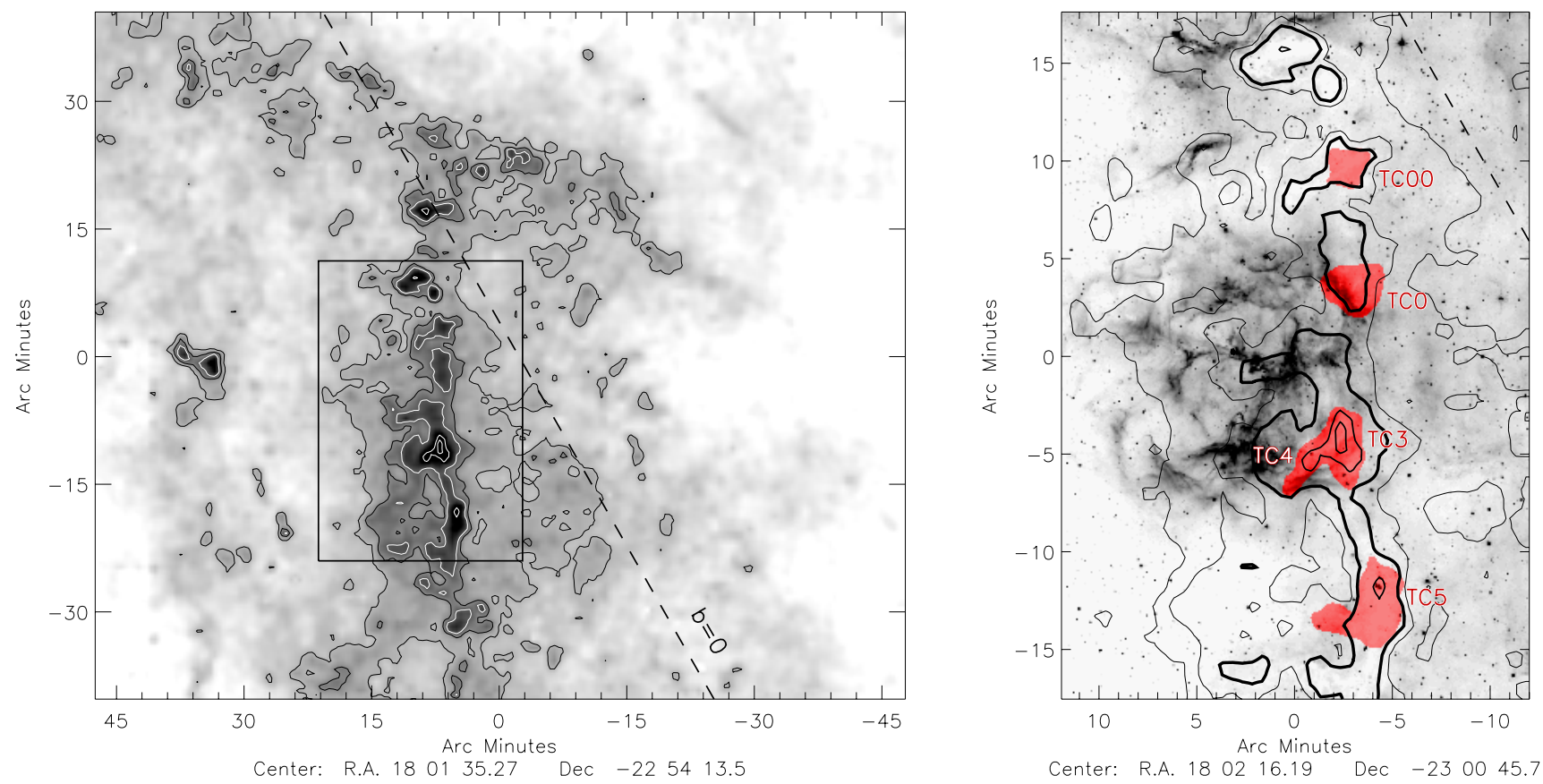

Fig. 6. Left: large scale extinction map of the Trifid region. Right: GLIMPSE $8 \mu \mathrm{m}$ image of the Trifid nebula with iso-extinction contours. In both figures contours correspond to $A_{V}=[10,15,20,40,60] \mathrm{mag}$, the dashed line follows the Galactic plane. The red shaded regions delineate the $S_{1.25}>10 \mathrm{mJy} / 11^{\prime \prime}$ beam Trifid Condensations (TC, Cernicharo et al. 1998) studied in Sect. 4.3 at 1 arcmin resolution.

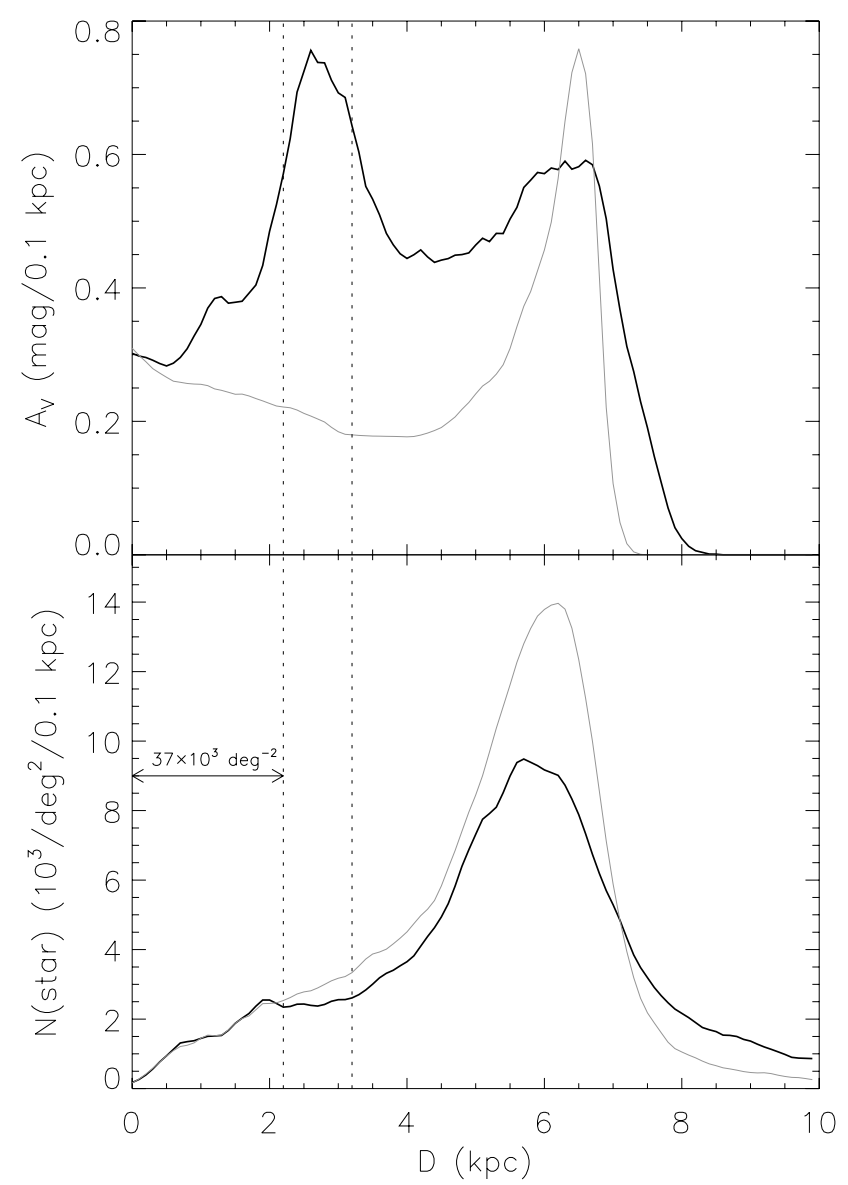

Fig. 7. Extinction (top) and star density (bottom) along the Trifid lineof-sight. The black line is the average value for the region where $A_{V}>20$ mag in Fig. 6; the gray line is for the region where $A_{V} \in$ $[0,1]$ mag. The dashed lines indicate the Trifid location at a distance of $2.7 \pm 0.5 \mathrm{kpc}$. where $\alpha$ is the angular size of a pixel in the map, $d$ is the distance to the cloud, $\mu$ the mean molecular weight corrected for the helium abundance, and $i$ represents a map pixel. With the dust-to-gas ratio proposed by Savage \& Mathis (1979), $N_{\mathrm{H}} / A_{V}=$ $1.87 \times 10^{21} \mathrm{~cm}^{-2} \mathrm{mag}^{-1}\left(N_{\mathrm{H}}=N_{H I}+N_{H_{2}}\right)$ and a distance of $2.7 \mathrm{kpc}$, we obtain a mass of $5.8 \times 10^{5} \mathrm{M}_{\odot}$. This value corresponds to twice the Orion (A+B) clouds mass (Cambrésy 1999; Wilson et al. 2005). Figure 8 shows how the integrated mass distribution varies with the extinction within the cloud. Regions with visual extinction greater than 20 mag account for less than $4 \%$ of the total mass of the cloud. The index of the cumulative mass distribution, $\alpha$, changes at $A_{V} \approx 25 \mathrm{mag}$ by a factor of 2 . With $M\left(A_{V}\right)$ being the mass enclosed in the contour of extinction $A_{V}$, we have:

$$
\begin{aligned}
& M\left(A_{V}\right)=M(0) \times 10^{-\alpha A_{V}} \\
& \text { with }\left\{\begin{array}{l}
\alpha=8.39 \times 10^{-2} \text { for } A_{V} \in[8,20] \\
\alpha=3.76 \times 10^{-2} \text { for } A_{V} \in[30,60] .
\end{array}\right.
\end{aligned}
$$

The variation of $\alpha$ is not a side-effect of the map combination technique nor the application of a corrected extinction law at large $A_{V}$ since the variation is also observed for a raw extinction map obtained only from the [3.6]-[4.5] color-excess and assuming a constant extinction law. It is however difficult to interpret the index variation as a consequence of the dust transition responsible of the extinction law evolution. The two events occurring at the same level may just be a coincidence. The $N_{\mathrm{H}} / A_{V}$ value is generally assumed to be universal (and shown recently by Planck collaboration 2011a, to be constant at large scale at all Galactic radii) although some authors suggested a decrease by a factor of 3 in deeply embedded regions (Winston et al. 2010). Dividing the mass estimate by any factor above a given extinction threshold would only produce an offset in Fig. 8, not a change of slope. A more complex evolution of $N_{\mathrm{H}} / A_{V}$ with the extinction would affect differently the Fig. 8 distribution but it seems unlikely to only produce a sharp slope change. 
L. Cambrésy et al.: Variation of the extinction law in the Trifid nebula

Table 3. Properties of the more extended Trifid cores detected at millimeter wavelength (Lefloch et al. 2008).

\begin{tabular}{lcccccccc}
\hline \hline $\begin{array}{l}\text { Name } \\
\text { [CLC98] }\end{array}$ & $\begin{array}{c}\text { Mass }^{a} \\
\left(M_{\odot}\right)\end{array}$ & $\begin{array}{c}\text { Mass }^{b} \\
\left(M_{\odot}\right)\end{array}$ & peak & $\begin{array}{c}A_{V}(\mathrm{mag}) \\
\text { median }\end{array}$ & outer & $\begin{array}{c}\text { Surface } \\
\left(\operatorname{arcmin}^{2}\right)\end{array}$ & $\begin{array}{c}\kappa \\
(\times)\end{array}$ & $\begin{array}{c}T_{\text {dust }} \\
(\mathrm{K})\end{array}$ \\
\hline TC00 & 936 & 911 & 27 & 22.7 & 20.0 & 3.5 & 1.3 & 13 \\
TC0 & 1607 & 1570 & 25 & 20.3 & 16.9 & 6.9 & 1.2 & 13 \\
TC3 & 3535 & 2783 & 78 & 40.4 & 27.6 & 7.3 & 2.6 & 8 \\
TC4 & 1149 & 945 & 52 & 32.8 & 23.7 & 3.0 & 2.7 & 8 \\
TC5 & 3543 & 3087 & 53 & 25.5 & 18.1 & 11.6 & 1.7 & 11 \\
\hline
\end{tabular}

Notes. Masses are expressed assuming a distance of $2.7 \mathrm{kpc}$. The mass from dust continuum emission is derived using $T_{\text {dust }}=15 \mathrm{~K}$ and $\kappa_{1.25}=$ $4.08 \times 10^{-1} \mathrm{~cm}^{2} \mathrm{gr}^{-1}$ (i.e. $C_{1.25}=7.336 \times 10^{-27} \mathrm{~cm}^{2} \mathrm{H}^{-1}$ ). By modifying these parameters the mass can be adjusted to the mass estimated from the dust extinction. The two last columns give these parameter values, providing only one of the two parameters is modified at the same time. ${ }^{(a)}$ Mass from the dust near-infrared extinction. ${ }^{(b)}$ Mass from the dust continuum millimeter emission.

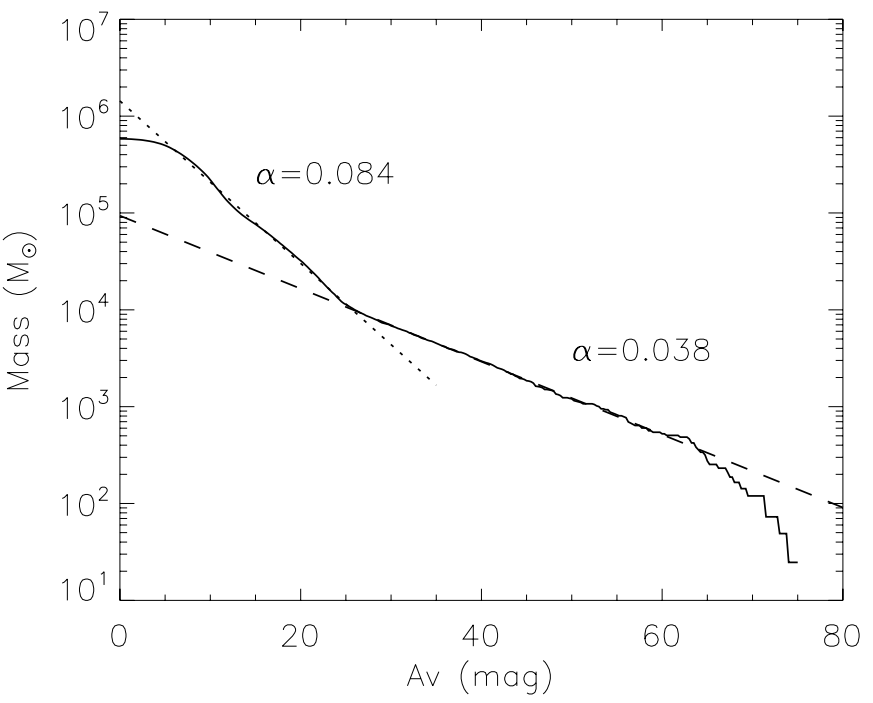

Fig. 8. Cumulative mass distribution for $d=2.7 \mathrm{kpc}$. The $\alpha$ index is defined in Eq. (2).

The millimeter wavelength emission from the cold dust continuum has been mapped by Cernicharo et al. (1998) and Lefloch et al. (2008). They have detected several dust condensations from the $1.25 \mathrm{~mm}$ emission. For simplicity we designate these dust condensations TC NN as in the original papers. This is fine in the Trifid context but obviously ambiguous in the general context and the full SIMBAD name is actually [CLC98] TC NN. For the more extended condensations we compare the mass from the dust infrared extinction and $1.25 \mathrm{~mm}$ emission. The results are presented in Table 3. The mass from extinction is obtained by integrating the area defined in the map at $1.25 \mathrm{~mm}$, convolved at 1 arcmin resolution, by the contour at $S_{1.25}=10 \mathrm{mJy} / 11^{\prime \prime}$ beam (red shaded area in Fig. 6). Because of the observational technique in the millimeter wavelengths, a spatial filtering removes the structure at scales larger than 4.5 arcmin in the dust continuum emission. To compare this map with the extinction map we scale it by adding the median extinction measured in the vicinity of each TC region. Basically the median extinction is measured in the area between the contours at $S_{1.25}=5$ and $10 \mathrm{mJy} / 11^{\prime \prime}$ around each condensation. The resulting offsets are given in the column labeled outer of Table 3. The mass from the map at $1.25 \mathrm{~mm}$ is derived assuming a dust temperature of $15 \mathrm{~K}$ and a dust opacity of $\kappa_{1.25}=4.08 \times 10^{-1} \mathrm{~cm}^{2} \mathrm{~g}^{-1}$ following the model B for $R_{V}=5.5$ by WD01. The corresponding extinction cross section per hydrogen atom is $C_{1.25}=7.336 \times 10^{-27} \mathrm{~cm}^{2} \mathrm{H}^{-1}$ after correction for the helium abundance and assuming a gas-to-dust ratio of 125 (Li \& Draine 2001). Lefloch et al. (2008) measured temperatures from 20 to $30 \mathrm{~K}$ using CO observations. The gas kinetic temperature and the dust temperature are however different in such regions and an arbitrary $T_{\text {dust }}=15 \mathrm{~K}$ is more appropriate. The masses we derived from the dust emission are systematically smaller. Two parameters, the temperature and the emissivity, can be adjusted to reach an agreement with the masses from the extinction. While TCO0 and T0 only need a small increase of the dust emissivity at $1.25 \mathrm{~mm}$, or a decrease of the temperature from 15 to $13 \mathrm{~K}$, TC3/4/5 require a more significant emissivity increase by a factor 1.7 to 2.7 . Using only the temperature as a free parameter would lead to estimates ranging from 11 to $8 \mathrm{~K}$. Such low temperatures have already been reported from submillimeter point sources (Désert et al. 2008; Planck collaboration 2011 b,c) observed by the Archeops experiment and by the Planck satellite. However, the condensations we are dealing with cover an extended field from 3 to $12 \operatorname{arcmin}^{2}$. Assuming a minimum temperature of $12 \mathrm{~K}$ for $\mathrm{TC} 3 / 4$, an emissivity enhancement by a factor 1.9 would make the mass estimates in agreement. This type of emissivity enhancement has been recently reported by Planck collaboration (2011d) in the Taurus. TC00 and TC 0 are starless condensations while TC3/4/5 form stars and undergo shocks. Our analysis suggest the dust emissivity at millimeter wavelength is enhanced for these condensations, especially for the densest ones. Complementary observations at longer wavelengths, from Herschel and Planck, will permit to better understand the physical properties of the interstellar medium in the Trifid region. We plan to follow up this study as soon as the data from the Herschel Infrared Galactic Plane Survey (Hi-GAL, Molinari et al. 2010b) are available.

\section{Conclusion}

We performed a detailed analysis of the extinction in the molecular cloud associated with the Trifid nebula. The direction and the distance of this cloud is a major difficulty compared to the other similar investigations published in the literature. To overcome this specificity we proposed an original method which combines the color-excess mapping with the color-color diagram analysis. The gain in sensitivity with this method allowed us to measure to extinction law at 3.6, 4.5 and $5.8 \mu \mathrm{m}$ and to unambiguously detect a variation at large absorption through an abrupt change of slope in the color-color plots. We interpreted this result as evidence for rapid dust evolution at high density, such as a new dust transition phase in the dense interstellar medium.

The extinction law parameters, $A_{\lambda} / A_{K_{\mathrm{s}}}$, are found to be larger in the very dense cores of the cloud, in agreement with dust models. Our values for $A_{V}>20 \mathrm{mag}$ are however larger than predicted by WD01 for dense regions $\left(R_{V}=5.5\right)$ and larger than previously reported in the literature at $3.6 \mu \mathrm{m}$. For $A_{V}<15 \mathrm{mag}$, 
which does still refer to dense material, our values match those predicted by the model remarkably well, for $R_{V}=5.5$, as expected. Our study is not sensitive to the diffuse medium for which $R_{V}=3.1$ with a typical visual extinction as low as $1 \mathrm{mag}$. Moreover, Zasowski et al. (2009) found a correlation of the extinction law for the diffuse interstellar medium with the galactocentric radius. They concluded the extinction curve follows the dust model with $R_{V}=5.5$ for a galactocentric radius consistent with the Trifid distance. It suggests the so-called diffuse medium already contains big grains in this part of the Galaxy. If the diffuse medium at the Trifid distance behaves like the dense medium in the solar neighborhood we can imagine the Trifid dense cores are also affected by the distance with a flatter extinction law.

Using our varying extinction law we built a composite extinction map of the Trifid nebula region. The maximum visual extinction is $80 \mathrm{mag}$ at 1 arcmin resolution. We have also investigated the matter distribution along the line-of-sight since several small clouds could have mimicked the presence of a single giant massive dark cloud. We found no significant contribution of other clouds in this direction and we were able to estimate the distance of the Trifid nebula at $2.7 \pm 0.5 \mathrm{kpc}$.

The Trifid molecular cloud is about twice the mass of the Orion molecular cloud. The comparison of the dust extinction and $1.25 \mathrm{~mm}$ continuum emission in the densest cores suggests the dust emissivity is probably enhanced by a factor of $2-3$ in these regions. This cloud appears as a young and massive version of the Orion or Rosette regions. The Trifid represents an earlier evolutionary status in the star-formation process with a first generation of OB stars but no significant embedded cluster yet. Several protostars in TC3/4 are likely the precursors of such clusters. Longer wavelength observations from Herschel and Planck are needed to better understand the dust properties and the interaction between the interstellar medium and the OB stars.

Acknowledgements. We thank the anonymous referee for his comments that help to clarify and improve this paper. We thank T. Dame for providing us with the $\mathrm{CO}$ datacube and for his assistance in using it. We are also grateful to A. Boogert for his help understanding the possible ice contributions to our analysis. This work is based in part on observations made with the Spitzer Space Telescope, which is operated by the Jet Propulsion Laboratory, California Institute of Technology under a contract with NASA. This work is based in part on data obtained as part of the UKIRT Infrared Deep Sky Survey. This publication makes use of data products from the Two Micron All Sky Survey, which is a joint project of the University of Massachusetts and the Infrared Processing and Analysis Center/California Institute of Technology, funded by the National Aeronautics and Space Administration and the National Science Foundation

\section{References}

Bergin, E. A., Melnick, G. J., Gerakines, P. A., Neufeld, D. A., \& Whittet, D. C. B. 2005, ApJ, 627, L33

Bernard, J. P., Abergel, A., Ristorcelli, I., et al. 1999, A\&A, 347, 640

Boogert, A. C. A., Huard, T. L., Cook, A. M., et al. 2011, ApJ, accepted [arXiv: 1101.2486]
Boulanger, F., Beichman, C., Desert, F. X., et al. 1988, ApJ, 332, 328 Cambrésy, L. 1999, A\&A, 345, 965

Cambrésy, L., Boulanger, F., Lagache, G., \& Stepnik, B. 2001, A\&A, 375, 999 Cambrésy, L., Beichman, C. A., Jarrett, T. H., \& Cutri, R. M. 2002, AJ, 123, 2559

Cambrésy, L., Jarrett, T. H., \& Beichman, C. A. 2005, A\&A, 435, 131

Casali, M., Adamson, A., Alves de Oliveira, C., et al. 2007, A\&A, 467, 777 Cernicharo, J., Lefloch, B., Cox, P., et al. 1998, Science, 282, 462

Chapman, N. L., Mundy, L. G., Lai, S., \& Evans, N. J. 2009, ApJ, 690, 496

Churchwell, E., Babler, B. L., Meade, M. R., et al. 2009, PASP, 121, 213

Dame, T. M., Hartmann, D., \& Thaddeus, P. 2001, ApJ, 547, 792

Désert, F., Macías-Pérez, J. F., Mayet, F., et al. 2008, A\&A, 481, 411

Dickman, R. L. 1978, AJ, 83, 363

Dobashi, K., Uehara, H., Kandori, R., et al. 2005, PASJ, 57, 1

Flaherty, K. M., Pipher, J. L., Megeath, S. T., et al. 2007, ApJ, 663, 1069

Hambly, N. C., Collins, R. S., Cross, N. J. G., et al. 2008, MNRAS, 384, 637

Hewett, P. C., Warren, S. J., Leggett, S. K., \& Hodgkin, S. T. 2006, MNRAS, 367,454

Hodgkin, S. T., Irwin, M. J., Hewett, P. C., \& Warren, S. J. 2009, MNRAS, 394, 675

Indebetouw, R., Mathis, J. S., Babler, B. L., et al. 2005, ApJ, 619, 931

Kohoutek, L., Mayer, P., \& Lorenz, R. 1999, A\&AS, 134, 129

Lada, C. J., Lada, E. A., Clemens, D. P., \& Bally, J. 1994, ApJ, 429, 694

Laureijs, R. J., Clark, F. O., \& Prusti, T. 1991, ApJ, 372, 185

Lawrence, A., Warren, S. J., Almaini, O., et al. 2007, MNRAS, 379, 1599

Lefloch, B., Cernicharo, J., \& Pardo, J. R. 2008, A\&A, 489, 157

Li, A., \& Draine, B. T. 2001, ApJ, 554, 778

Lombardi, M. 2009, A\&A, 493, 735

Lombardi, M., \& Alves, J. 2001, A\&A, 377, 1023

Lynds, B. T., Canzian, B. J., \& Oneil, Jr., E. J. 1985, ApJ, 288, 164

Marshall, D. J., Robin, A. C., Reylé, C., Schultheis, M., \& Picaud, S. 2006, A\&A, 453, 635

Marshall, D. J., Joncas, G., \& Jones, A. P. 2009, ApJ, 706, 727

McClure, M. 2009, ApJ, 693, L81

McMillan, P. J., \& Binney, J. J. 2010, MNRAS, 402, 934

Molinari, S., Swinyard, B., Bally, J., et al. 2010a, A\&A, 518, L100

Molinari, S., Swinyard, B., Bally, J., et al. 2010b, PASP, 122, 314

Nishiyama, S., Nagata, T., Kusakabe, N., et al. 2006, ApJ, 638, 839

Olofsson, S., \& Olofsson, G. 2010, A\&A, 522, A84

Planck collaboration 2011a [arXiv: 1101.2032]

Planck collaboration 2011b [arXiv: 1101.2035]

Planck collaboration 2011c [arXiv: 1101.2034]

Planck collaboration 2011d [arXiv: 1101.2037]

Rho, J., Corcoran, M. F., Chu, Y., \& Reach, W. T. 2001, ApJ, 562, 446

Rho, J., Ramírez, S. V., Corcoran, M. F., Hamaguchi, K., \& Lefloch, B. 2004, ApJ, 607, 904

Rho, J., Reach, W. T., Lefloch, B., \& Fazio, G. G. 2006, ApJ, 643, 965

Rho, J., Lefloch, B., Reach, W. T., \& Cernicharo, J. 2008, in Handbook of Star Forming Regions, vol II, ASP Conf. Ser., 5, 509

Rieke, G. H., \& Lebofsky, M. J. 1985, ApJ, 288, 618

Robin, A. C., Reylé, C., Derrière, S., \& Picaud, S. 2003, A\&A, 409, 523

Román-Zúñiga, C. G., Lada, C. J., Muench, A., \& Alves, J. F. 2007, ApJ, 664, 357

Savage, B. D., \& Mathis, J. S. 1979, ARA\&A, 17, 73

Skrutskie, M. F., Cutri, R. M., Stiening, R., et al. 2006, AJ, 131, 1163

Vallée, J. P. 2008, AJ, 135, 1301

Weingartner, J. C., \& Draine, B. T. 2001, ApJ, 548, 296

Whittet, D. C. B., Gerakines, P. A., Tielens, A. G. G. M., et al. 1998, ApJ, 498, L159

Whittet, D. C. B., Gerakines, P. A., Hough, J. H., \& Shenoy, S. S. 2001, ApJ, 547,872

Wilson, B. A., Dame, T. M., Masheder, M. R. W., \& Thaddeus, P. 2005, A\&A, 430,523

Winston, E., Megeath, S. T., Wolk, S. J., et al. 2010, AJ, 140, 266

Zasowski, G., Majewski, S. R., Indebetouw, R., et al. 2009, ApJ, 707, 510 